\title{
Solving Integral Equations by Means of Fixed Point Theory
}

\author{
E. Karapinar $\mathbb{D},{ }^{1,2,3}$ A. Fulga $\mathbb{D},{ }^{4}$ N. Shahzad $\mathbb{D},{ }^{5}$ and A. F. Roldán López de Hierro $\mathbb{D}^{6}$ \\ ${ }^{1}$ Division of Applied Mathematics, Thu Dau Mot University, Binh Duong Province, Vietnam \\ ${ }^{2}$ Department of Medical Research, China Medical University Hospital, China Medical University, 40402, Taichung, Taiwan \\ ${ }^{3}$ Department of Mathematics, Çankaya University, 06790, Etimesgut, Ankara, Turkey \\ ${ }^{4}$ Department of Mathematics and Computer Sciences, Universitatea Transilvania Brasov, Brasov, Romania \\ ${ }^{5}$ Department of Mathematics, Faculty of Science, King Abdulaziz University, P.O.B. 80203, Jeddah 21589, Saudi Arabia \\ ${ }^{6}$ Department of Statistics and Operations Research, University of Granada, Granada, Spain
}

Correspondence should be addressed to E. Karapinar; erdalkarapinar@tdmu.edu.vn

and A. F. Roldán López de Hierro; aroldan@ugr.es

Received 13 September 2021; Accepted 29 December 2021; Published 10 February 2022

Academic Editor: Feliz Minhos

Copyright @ 2022 E. Karapínar et al. This is an open access article distributed under the Creative Commons Attribution License, which permits unrestricted use, distribution, and reproduction in any medium, provided the original work is properly cited.

\begin{abstract}
One of the most interesting tasks in mathematics is, undoubtedly, to solve any kind of equations. Naturally, this problem has occupied the minds of mathematicians since the dawn of algebra. There are hundreds of techniques for solving many classes of equations, facing the problem of finding solutions and studying whether such solutions are unique or multiple. One of the recent methodologies that is having great success in this field of study is the fixed point theory. Its iterative procedures are applicable to a great variety of contexts in which other algorithms fail. In this paper, we study a very general class of integral equations by means of a novel family of contractions in the setting of metric spaces. The main advantage of this family is the fact that its general contractivity condition can be particularized in a wide range of ways, depending on many parameters. Furthermore, such a contractivity condition involves many distinct terms that can be either adding or multiplying between them. In addition to this, the main contractivity condition makes use of the self-composition of the operator, whose associated theorems used to be more general than the corresponding ones by only using such mapping. In this setting, we demonstrate some fixed point theorems that guarantee the existence and, in some cases, the uniqueness, of fixed points that can be interpreted as solutions of the mentioned integral equations.
\end{abstract}

\section{Introduction}

Nowadays, nonlinear analysis is one of the most active branches of mathematics. Its applications to real-life contexts have attained great success. Physics, engineering, chemistry, biology, and economy are some of the scientific areas that have benefited the most from the techniques developed in nonlinear analysis. In this context, fixed point theory has played an important role in the development of new methodologies for the determination of solutions of certain equations of several types, such as matrix equations, integral equations, and differential equations.

In principle, the elements used by fixed point theory are few and very simple to handle a nonlinear operator for which we want to find its possible fixed points, a real metric that endows the underlying space with a complete character, and an inequality (called the contractivity condition) that is strong enough to ensure the existence of fixed points. With these three ingredients, it is possible to propose good fixed point theorems, as has been done for the last seventy years (see, for instance, Boyd and Wong [1], Caristi [2], Chatterjea [3], Hardy and Rogers [4], Kannan [5, 6], Ćirić [7], Geragthy [8], Meir and Keeler [9], Samet et al. [10], Khojasteh et al. [11], Kutbi et al. [12], and Jleli and Samet [13]).

Based on these three initial tools, the possibilities that this field of study has shown have been practically endless. On the one hand, researchers have worked with increasingly abstract metric spaces. In some of these cases, the object associated with the distance between two points has not been a single real number but much more general abstract objects. On the other hand, the operators involved in these studies 
have been increasingly general, including the possibility of studying multidimensional fixed points (see [14]). Finally, the contractivity condition is the part that has received the most attention within the field of fixed point theory.

In recent times, major efforts have been done in order to introduce as weak as possible contractivity conditions. For instance, it is usual to find auxiliary functions that help to consider extremely weak inequalities. Having this aim in mind, we would like to highlight here two possible extensions.

(i) On the one hand, although the first contractivity conditions only considered a small quantity of terms, after the appearance of the Cirić theorem [7], the current versions involve more and more terms in their developments. This is the case, for instance, of Karapnar's interpolative-type contractions [15], but many other results can be cited in this line of research (see $[16,17])$

(ii) On the other hand, in general, notice that the good and reasonable properties that an operator $T: M$ $\longrightarrow M$ satisfies are usually inherited by the selfcomposition $T^{2}=T \circ T$, but it is possible that $T^{2}$ enjoys those good properties without $T$ doing it. This is the case, for instance, of continuity: it is possible for $T^{2}$ to be continuous without $T$ being continuous. In this sense, some results (like Istrățescu's fixed point theorem; see $[18,19])$ employing $T^{2}$ are more general than their corresponding ones with $T$

One of the powerful applications of fixed point theory can be found in the context of integral equations, whose recent numerical treatments have made great scientific advances in this field (see, for instance, collocation methods [20], operational matrix methods [21-23], Galerkin methods [24, 25], and Krylov subspace methods [26]).

In this paper, we introduce a new family of contractive mappings that we call hybrid-interpolative Reich-Istrățescutype contractions because they are inspired by the previous classes of contractive operators. The main advantage of this new family of contractive mappings is that they allow us to present, at the same time, contractivity conditions that involve a large number of terms, including some with the selfcomposition $T^{2}$ of the operator, and which are placed either adding or multiplying to the other terms. Furthermore, we introduce some fixed point results that confirm that this kind of operators is appropriate in this field of study. Finally, we illustrate the utility of the novel theorems by introducing a novel application in the setting of integral equations.

This work is organized as follows. Section 2 is dedicated to presenting some notations, preliminaries, and related results in the field of fixed point theory. In Section 3, we describe a complete study about the behavior of the Picard sequences that we will handle in the following sections. The main results of this paper can be found in the Section 4, and direct consequences are placed in Section 5. The application of the main statements is developed in Section 6 . Finally, some conclusions and prospect works are discussed in Section 7.

\section{Background on Fixed Point Theory}

In this work, we denote by $\mathbb{R}$ and $\mathbb{N}=\{0,1,2, \cdots\}$ the set of all real numbers and the set of nonnegative integers, respectively. Let $(M, d)$ be a metric space and let $T: M$ $\longrightarrow M$ be a mapping. A point $u \in M$ is a fixed point of $T$ if $T u=u$. We will denote by $* \operatorname{Fix}_{T}(M)$ the set of all fixed points of $T$ in $M$.

Given $n \in \mathbb{N}$, the mapping $T^{n}=T \circ T \circ \ldots(n) \circ T: M$ $\longrightarrow M$ is the $n$-th iterate of $T$ (as convention, we agree that $T^{0}$ is the identity mapping on $M$ ). Given $z_{0} \in M$, the sequence $\left\{z_{n}\right\}_{n \in \mathbb{N}}$ defined by $z_{n}=T^{n} z_{0}$ for all $n \in \mathbb{N}$ is the Picard sequence of $T$ based on $z_{0}$. Such sequence can recursively be defined as $z_{n+1}=T z_{n}$ for all $n \in \mathbb{N}$. A mapping $T$ is called a Picard operator if each Picard sequence of such operator converges to one of its fixed points.

A binary relation on the set $M$ is a nonempty subset $\mathscr{R}$ of the Cartesian product $M \times M$. We will write $z \mathscr{R} t$ when two points $z, t \in M$ verify $(u, t) \in \mathscr{R}$.

Definition 1. Let $\alpha: M \times M \longrightarrow[0, \infty)$ be a function. A mapping $T: M \longrightarrow M$ such that

$$
\text { if } z \in M, \alpha(z, T z) \geq 1 \Rightarrow \alpha\left(T z, T^{2} z\right) \geq 1
$$

is known as an $\alpha$-orbital admissible mapping.

One of the first results in fixed point theory in which the contractivity condition was stated in terms of its selfcomposition $T^{2}=T \circ T$ rather than in terms of $T$ was due to Istrățescu (see $[18,19])$.

Theorem 2 (Istrățescu $[18,19])$. Given a complete metric space $(M, d)$, every continuous map $T: M \longrightarrow M$ is a Picard operator provided that there exist $a, c \in(0,1)$ such that $a+c$ $<1$ and

$$
d\left(T^{2} z, T^{2} t\right) \leq a d(T z, T t)+c d(z, t)
$$

for all $z, t \in M$.

Notice that the good properties (like continuity) of an operator $T$ are usually inherited by $T^{2}$, but it is possible that $T^{2}$ enjoys those good properties without $T$ doing it. In this sense, some results employing $T^{2}$ are more general that their corresponding ones with $T$. Some generalizations of this result in different abstract metric spaces ( $b$-metric spaces, ordered metric spaces, cone metric spaces, etc.) were presented in recent papers (see [27-29]).

In other lines of research, inspired by Kannan's theorem [5, 6], Karapnar introduced in [15] a family of contractions in which the distances of the right-hand side of the contractivity condition are multiplying instead of adding up. Also, notice that his contractivity condition must only be verified by pairs of points in the metric space that are not fixed points of the considered nonlinear operator, which avoids any kind of indetermination of the involved powers. 
Theorem 3 (Karapnar [15]). Let $(M, d)$ be a complete metric space and let $T: M \longrightarrow M$ be a mapping such that there exist constants $k \in 0,1)$ and $\lambda \in(0,1)$ satisfying

$$
d(T z, T t) \leq k \cdot d(z, T z)^{\lambda} \cdot d(t, T t)^{1-\lambda}
$$

for all $z, t \in M \backslash * \operatorname{Fix}_{T}(M)$. Then, $T$ has a unique fixed point in $M$.

In the previous result, as $z$ and $t$ are not fixed points of $T$ , then $d(z, T z)>0$ and $d(t, T t)>0$. Furthermore, as $\lambda>0$ and $1-\lambda>0$, then the expressions $d(z, T z)^{\lambda}$ and $d$ $(t, T t)^{1-\lambda}$ are well defined. However, in the main results that we will introduce later, we will employ expressions such as

$$
a_{4} d(T z, T t)^{\lambda}
$$

which we would like to explain for the sake of clarity: on the one hand, (4) means the product $a_{4} \cdot[d(T z, T t)]^{\lambda}$, where $a_{4}$ and $\lambda$ are nonnegative real numbers (notice that the exponent $\lambda$ only affects the distance $d(T z, T t)$, and we avoid to write the brackets); on the other hand, the power $d$ $(T z, T t)^{\lambda}$ is not well defined when the base and the exponent take the value 0 at the same time. However, for our purposes, we must advise the reader that, when the base and the exponent are 0 at the same time, we will use the convention $0^{0}=1$

\section{Study of the Behavior of Some Picard Sequences}

In this section, we describe the behavior of some sequences that will be of importance in the proofs of the main results of this work.

Proposition 4. Given $c \in[0, \infty)$, let $\left\{r_{n}\right\} \subset[0, \infty)$ be a sequence such that

$$
r_{n+2} \leq c \cdot \max \left\{r_{n}, r_{n+1}\right\} \text { for all } n \in \mathbb{N} \text {. }
$$

Let $\Delta=\max \left\{r_{0}, r_{1}\right\}$. Then,

$$
r_{2 n} \leq c^{n} \Delta, r_{2 n+1} \leq c^{n} \Delta \text { for all } n \geq 1,
$$

which is equivalent to

$$
r_{n+2} \leq c^{* E[n / 2]} \cdot \Delta \text { for all } n \in \mathbb{N}
$$

In particular, if $c \in[0,1)$, then $\left\{r_{n}\right\} \longrightarrow 0$.

Proof. For $n=0$ in (5),

$$
r_{2} \leq c \cdot \max \left\{r_{0}, r_{1}\right\}=c \Delta
$$

and for $n=1$ in (5)

$$
\begin{aligned}
r_{3} & \leq c \cdot \max \left\{r_{1}, r_{2}\right\} \leq c \cdot \max \left\{r_{1}, c \cdot \max \left\{r_{0}, r_{1}\right\}\right\} \\
& =c \cdot \max \left\{r_{1}, c r_{0}, c r_{1}\right\} \leq c \cdot \max \left\{r_{1}, c r_{0}\right\} \\
& \leq c \cdot \max \left\{r_{1}, r_{0}\right\}=c \Delta .
\end{aligned}
$$

This means that inequalities (6) hold for $n=1$. Suppose that (6) holds for some $n \in \mathbb{N}$, that is,

$$
r_{2 n} \leq \mathrm{c}^{n} \Delta, r_{2 n+1} \leq \mathrm{c}^{n} \Delta
$$

Therefore,

$$
\begin{gathered}
r_{2 n+2} \leq c \cdot \max \left\{r_{2 n}, r_{2 n+1}\right\} \leq c \cdot \max \left\{c^{n} \Delta, c^{n} \Delta\right\}=c^{n+1} \Delta, \\
r_{2 n+3} \leq c \cdot \max \left\{r_{2 n+1}, r_{2 n+2}\right\} \leq c \cdot \max \left\{c^{n} \Delta, c^{n+1} \Delta\right\}=c^{n+1} \Delta .
\end{gathered}
$$

This completes the induction.

Lemma 5. Let $\left\{z_{n}\right\}_{n \in \mathbb{N}}$ be a sequence on a metric space $(M, d)$. Suppose that there is $c \in[0,1)$ such that

$d\left(z_{n+2}, z_{n+3}\right) \leq c \cdot \max \left\{d\left(z_{n}, z_{n+1}\right), d\left(z_{n+1}, z_{n+2}\right)\right\}$ for all $n \in \mathbb{N}$.

Then, $\left\{z_{n}\right\}_{n \in \mathbb{N}}$ is a Cauchy sequence in $(M, d)$.

Proof. Let us consider the sequence $\left\{r_{n}\right\}$ defined by $r_{n}=d($ $\mathrm{z}_{n}, \mathrm{z}_{n+1}$ ) for all $n \in \mathbb{N}$. By the hypothesis, this sequence verifies (5). Then, Proposition 4 guarantees that

$$
d\left(z_{2 n}, z_{2 n+1}\right)=r_{2 n} \leq c^{n} \Delta, d\left(z_{2 n+1}, z_{2 n+2}\right)=r_{2 n+1} \leq c^{n} \Delta \text { for all } n \geq 1,
$$

where $\Delta=\max \left\{r_{0}, r_{1}\right\}$. In particular,

$$
d\left(z_{2 n}, z_{2 n+1}\right)+d\left(z_{2 n+1}, z_{2 n+2}\right) \leq 2 c^{n} \Delta \text { for all } n \geq 1
$$

If $c=0$ or $\Delta=0$, then $\left\{z_{n}\right\}_{n \geq 2}$ is a constant sequence, so it is a Cauchy sequence. Suppose that $c>0$ and $\Delta>0$. In order to prove that the sequence $\left\{z_{n}\right\}_{n \in \mathbb{N}}$ is a Cauchy sequence in $(M, d)$, let $\varepsilon>0$ be arbitrary. Since $\varepsilon /(2 \Delta)>0$ and $0<c<1$, there is a natural number $n_{0}>1$ such that

$$
\sum_{k=n_{0}}^{+\infty} c^{k}<\frac{\varepsilon}{2 \Delta}
$$

In particular,

$2 \Delta \sum_{k=n_{0}}^{p} c^{k}<2 \Delta \sum_{k=n_{0}}^{+\infty} c^{k}<\varepsilon$ for all $p \in \mathbb{N}$ such that $p \geq n_{0}$. 
Let $n, m \in \mathbb{N}$ such that $m>n \geq 2 n_{0}$. Let $p$ be a natural number such that $p \geq n_{0}+1$ and $2 p \geq m$. Therefore, by (14),

$$
\begin{aligned}
d\left(z_{n}, z_{m}\right) & \leq \sum_{j=n}^{m-1} d\left(z_{j}, z_{j+1}\right) \leq \sum_{j=2 n_{0}}^{2 p-1} d\left(z_{j}, z_{j+1}\right) \\
& =\sum_{k=n_{0}}^{p-1}\left[d\left(z_{2 k}, z_{2 k+1}\right)+d\left(z_{2 k+1}, z_{2 k+2}\right)\right] \\
& \leq \sum_{k=n_{0}}^{p-1} 2 c^{k} \Delta \leq 2 \Delta \sum_{k=n_{0}}^{p} c^{k}<2 \Delta \sum_{k=n_{0}}^{+\infty} c^{k}<\varepsilon .
\end{aligned}
$$

This proves that $\left\{z_{n}\right\}_{n \in \mathbb{N}}$ is a Cauchy sequence in $(M, d)$

Remark 6. Taking into account that, in general, the notation $\Delta^{\alpha^{n}}$ is not well defined because the number $\Delta^{\left(\alpha^{n}\right)}$ is distinct to $\left(\Delta^{\alpha}\right)^{n}$, we clarify that, in the next statement, we use the convention:

$$
\Delta^{\alpha^{n}}=\Delta^{\left(\alpha^{n}\right)}
$$

Proposition 7. Given $c \in[0, \infty)$ and $\alpha \in(0,1)$, let $\left\{r_{n}\right\} \subset[0$, $\infty)$ be a sequence such that

$$
r_{n+2} \leq c \cdot \max \left\{r_{n}, r_{n+1}\right\}^{\alpha} \text { for all } n \in \mathbb{N}
$$

Let $\Delta=\max \left\{r_{0}, r_{1}, 1\right\}$. Then,

$r_{2 n} \leq c^{1+\alpha+\alpha^{2}+\cdots+\alpha^{n-1}} \Delta^{\alpha^{n}}, r_{2 n+1} \leq c^{1+\alpha+\alpha^{2}+\cdots+\alpha^{n-1}} \Delta^{\alpha^{n}}$ for all $n \geq 1$.

Therefore,

$$
\limsup _{n \longrightarrow+\infty} r_{n} \leq c^{1 /(1-\alpha)}
$$

Proof. If $c=0$, the announced properties are trivial. Suppose that $c>0$. Since $\alpha \in(0,1)$, we know that

$\alpha^{m+1}<\alpha^{m}<\alpha^{m-1}<\cdots<\alpha^{2}<\alpha$ for all $m \in \mathbb{N}, m \geq 1$.

Therefore, as $\Delta \geq 1$, then

$$
\Delta^{\alpha^{m+1}}<\Delta^{\alpha^{m}}<\Delta^{\alpha^{m-1}}<\cdots<\Delta^{\alpha^{2}}<\Delta^{\alpha} \text { for all } m \in \mathbb{N}, m \geq 1 .
$$

Using $n=0$ in (19),

$$
r_{2} \leq c \cdot \max \left\{r_{0}, r_{1}\right\}^{\alpha} \leq c \Delta^{\alpha}
$$

and if $n=1$ in (19), using (23),

$$
\begin{aligned}
r_{3} & \leq c \cdot \max \left\{r_{1}, r_{2}\right\}^{\alpha} \leq c \cdot \max \left\{\Delta, c \Delta^{\alpha}\right\}^{\alpha} \\
& =c \cdot \max \left\{\Delta^{\alpha}, c^{\alpha} \Delta^{\alpha^{2}}\right\} \leq c \cdot \max \left\{\Delta^{\alpha}, c^{\alpha} \Delta^{\alpha}\right\} \\
& =c \Delta^{\alpha} \cdot \max \left\{1, c^{\alpha}\right\}=c \Delta^{\alpha} .
\end{aligned}
$$

The previous two inequalities mean that (20) holds for $n=1$. Suppose that (20) is fulfilled for some $n \in \mathbb{N}$, and we are going to prove it for $n+1$. Indeed,

$$
\begin{aligned}
r_{2 n+2} & \leq c \cdot \max \left\{r_{2 n}, r_{2 n+1}\right\}^{\alpha} \\
& \leq c \cdot \max \left\{c^{1+\alpha+\alpha^{2}+\cdots+\alpha^{n-1}} \Delta^{\alpha^{n}}, c^{1+\alpha+\alpha^{2}+\cdots+\alpha^{n-1}} \Delta^{\alpha^{n}}\right\}^{\alpha} \\
& =c \cdot\left(c^{1+\alpha+\alpha^{2}+\cdots+\alpha^{n-1}} \Delta^{\alpha^{n}}\right)^{\alpha}=c \cdot\left(c^{\alpha+\alpha^{2}+\cdots+\alpha^{n-1}+\alpha^{n}} \Delta^{\alpha^{n+1}}\right) \\
& =c^{1+\alpha+\alpha^{2}+\cdots+\alpha^{n-1}+\alpha^{n}} \Delta^{\alpha^{n+1}},
\end{aligned}
$$

and using (23),

$$
\begin{aligned}
r_{2 n+3} & \leq c \cdot \max \left\{r_{2 n+1}, r_{2 n+2}\right\}^{\alpha} \\
& \leq c \cdot \max \left\{c^{1+\alpha+\alpha^{2}+\cdots+\alpha^{n-1}} \Delta^{\alpha^{n}}, c^{1+\alpha+\alpha^{2}+\cdots+\alpha^{n-1}+\alpha^{n}} \Delta^{\alpha^{n+1}}\right\}^{\alpha} \\
& =c \cdot \max \left\{c^{\alpha+\alpha^{2}+\cdots+\alpha^{n-1}+\alpha^{n}} \Delta^{\alpha^{n+1}}, c^{\alpha+\alpha^{2}+\cdots+\alpha^{n-1}+\alpha^{n}+\alpha^{n+1}} \Delta^{\alpha^{n+2}}\right\} \\
& \leq c \cdot \max \left\{c^{\alpha+\alpha^{2}+\cdots+\alpha^{n-1}+\alpha^{n}} \Delta^{\alpha^{n+1}}, c^{\alpha+\alpha^{2}+\cdots+\alpha^{n-1}+\alpha^{n}+\alpha^{n+1}} \Delta^{\alpha^{n+1}}\right\} \\
& =c \Delta^{\alpha^{n+1}} \cdot \max \left\{c^{\alpha+\alpha^{2}+\cdots+\alpha^{n-1}+\alpha^{n}}, c^{\alpha+\alpha^{2}+\cdots+\alpha^{n-1}+\alpha^{n}+\alpha^{n+1}}\right\} \\
& =c \Delta^{\alpha^{n+1}} \cdot c^{\alpha+\alpha^{2}+\cdots+\alpha^{n-1}+\alpha^{n}}=c^{1+\alpha+\alpha^{2}+\cdots+\alpha^{n-1}+\alpha^{n}} \Delta^{\alpha^{n+1}},
\end{aligned}
$$

which completes the induction. Then, (20) holds.

Taking into account that $\left\{\alpha^{n}\right\}_{n \in \mathbb{N}} \longrightarrow 0$, we know that $\left\{\Delta^{\alpha^{n}}\right\}_{n \in \mathbb{N}} \longrightarrow \Delta^{0}=1$. On the other hand,

$$
\lim _{n \longrightarrow+\infty}\left(1+\alpha+\alpha^{2}+\cdots+\alpha^{n-1}+\alpha^{n}\right)=\sum_{k=0}^{+\infty} \alpha^{k}=\frac{1}{1-\alpha}
$$

then

$$
\lim _{n \longrightarrow+\infty} c^{1+\alpha+\alpha^{2}+\cdots+\alpha^{n-1}+\alpha^{n}}=c^{1 /(1-\alpha)} .
$$

Hence,

$$
\limsup _{n \rightarrow+\infty} r_{n} \leq c^{1 /(1-\alpha)}
$$

In order to check how different the conditions (19), where $\alpha \in(0,1)$, and (5), where $\alpha=1$, are, let us consider the following example. 
Example 8. Let $\left\{r_{n}\right\}_{n \in \mathbb{N}} \subset(0, \infty)$ be the sequence defined by

$$
r_{1}=r_{2}=0.25, r_{n+2}=c \cdot \max \left\{r_{n}, r_{n+1}\right\}^{\alpha} \text { for all } n \in \mathbb{N} \text {, }
$$

where $c=\alpha=0.5$. Then, it can be easily proven by induction that $\left\{r_{n}\right\}$ is the constant sequence given by $r_{n}=0.25$ for all $n \in \mathbb{N}$. Indeed, if $r_{n}=r_{n+1}=0.25$ for some $n \in \mathbb{N}$, then

$$
r_{n+2}=c \cdot \max \left\{r_{n}, r_{n+1}\right\}^{\alpha}=0.5 \cdot \max \{0.25,0.25\}^{0.5}=0.25
$$

As a consequence, $\left\{r_{n}\right\} \longrightarrow 0.25$, but it does not converge to zero, as in Proposition 4.

Corollary 9. Let $\left\{z_{n}\right\}_{n \in \mathbb{N}}$ be a sequence on a metric space $M, d)$. Suppose that there are $c \in[0,1)$ and $\alpha, \beta \in[0,1]$ verifying $\alpha+\beta=1$ such that

$$
d\left(z_{n+2}, z_{n+3}\right) \leq c \cdot d\left(z_{n}, z_{n+1}\right)^{\alpha} \cdot d\left(z_{n+1}, z_{n+2}\right)^{\beta} \text { for all } n \in \mathbb{N}
$$

Then, $\left\{z_{n}\right\}_{n \in \mathbb{N}}$ is a Cauchy sequence in $(M, d)$.
Proof. Notice that, for all $n \in \mathbb{N}$,

$$
\begin{aligned}
\mathrm{d}\left(z_{n+2}, z_{n+3}\right) \leq & c \cdot d\left(z_{n}, z_{n+1}\right)^{\alpha} \cdot d\left(z_{n+1}, z_{n+2}\right)^{\beta} \\
\leq & c \cdot \max \left\{d\left(z_{n}, z_{n+1}\right), d\left(z_{n+1}, z_{n+2}\right)\right\}^{\alpha} \\
& \cdot \max \left\{d\left(z_{n}, z_{n+1}\right), d\left(z_{n+1}, z_{n+2}\right)\right\}^{\beta} \\
= & c \cdot \max \left\{d\left(z_{n}, z_{n+1}\right), d\left(z_{n+1}, z_{n+2}\right)\right\}^{\alpha+\beta} \\
= & c \cdot \max \left\{d\left(z_{n}, z_{n+1}\right), d\left(z_{n+1}, z_{n+2}\right)\right\}
\end{aligned}
$$

Then, Lemma 5 is applicable.

\section{Fixed Point Theorems for Hybrid- Interpolative Reich-Istrăţescu- Type Contractions}

In this section, we introduce the novel class of contractive mappings based on Reich and Istrățescu's approaches.

Definition 10. Let $(M, d)$ be a metric space and let $\alpha: M \times$ $M \longrightarrow[0, \infty)$ be a function. A mapping $T: M \longrightarrow M$ is a hybrid-interpolative Reich-Istrățescu-type contraction in the case that for some $\lambda \in 0, \infty)$, there exist a constant $k \in 0$ , 1) and six numbers $a_{1}, a_{2}, a_{3}, a_{4}, a_{5}, \delta \geq 0$ such that, for all distinct $z, t \in M \backslash * \operatorname{Fix}_{T}(M)$,

$$
\alpha(z, t) d\left(T^{2} z, T^{2} t\right) \leq k \cdot \mathscr{I}^{\lambda}(z, t),
$$

where

$$
\mathscr{I}^{\lambda}(z, t)= \begin{cases}{\left[a_{1} d(z, t)^{\lambda}+a_{2} d(z, T z)^{\lambda}+a_{3} d(t, T t)^{\lambda}+a_{4} d(T z, T t)^{\lambda}+a_{5} d\left(T z, T^{2} z\right)^{\lambda}+\delta d\left(T t, T^{2} t\right)^{\lambda}\right]^{1 / \lambda},} & \text { for } \lambda>0, \\ d(z, t)^{a_{1}} \cdot d(z, T z)^{a_{2}} \cdot d(t, T t)^{a_{3}} \cdot d(T z, T t)^{a_{4}} \cdot d\left(T z, T^{2} z\right)^{a_{5}} \cdot d\left(T t, T^{2} t\right)^{\delta}, & \text { for } \lambda>0, \\ \sum_{i=1}^{5} a_{i}+\delta \leq 1, \text { if } \lambda>0, \sum_{i=1}^{5} a_{i}+\delta=1, \text { if } \lambda=0 . & \end{cases}
$$

\section{Remark 11}

(1) As we have commented in the second section, when $\lambda>0$, the expression $d(z, t)^{\lambda}$ is well defined even if the base is zero. However, although $z \neq t$ in (36), in the case $\lambda=0$, it is possible that we can find the indetermination $0^{0}$ in the expression of $\mathscr{I}^{\lambda}(z, t)$. In such a case, we will use the convention $0^{0}=1$ to avoid such indetermination. In other words, if some exponent in the expression of $\mathscr{I}^{\lambda}(z, t)$ is zero, then its correspondent power will take the value 1 . Notice that in this case, it is impossible that all exponents are zero because $\sum_{i=1}^{5} a_{i}+\delta=1$
(2) We can believe that the cases

$$
\sum_{i=1}^{5} a_{i}+\delta \leq 1, \sum_{i=1}^{5} a_{i}+\delta=1
$$

are equivalent because if $\sum_{i=1}^{5} a_{i}+\delta<1$, then we can replace $\delta$ by $\left.\delta^{\prime} \in 0,1\right)$ such that $\sum_{i=1}^{5} a_{i}+\delta^{\prime}=1$, and the mapping $T$ is also a hybrid-interpolative ReichIstrățescu-type contraction by considering the new parameters $a_{1}, a_{2}, a_{3}, a_{4}, a_{5}, \delta^{\prime} \geq 0$. However, as we will show later, when $\lambda=0$, we cannot permit the sum of the exponents to be less than 1 because, in 
such case, the sequence of distances between a term and its consecutive might not converge to zero (so it is not Cauchy)

(3) Although the definition of $\mathscr{I}^{\lambda}(z, t)$, for $\lambda>0$, is very different to the definition of $\mathscr{I}^{0}(z, t)(\lambda=0)$ because the first case uses additions and the second case involves products, there is a particular case in which both algebraic expressions lead to the same contractivity condition. It corresponds to the choice:

$$
a_{1}=1, a_{2}=a_{3}=a_{4}=a_{5}=\delta=0 \text {. }
$$

In this case, if $\lambda>0$,

$$
\mathscr{I}^{\lambda}(z, t)=\left[1 \cdot d(z, t)^{\lambda}\right]^{1 / \lambda}=d(z, t)
$$

and if $\lambda=0$,

$$
\begin{aligned}
\mathscr{I}^{0}(z, t)= & d(z, t)^{1} \cdot d(z, T z)^{0} \cdot d(t, T t)^{0} \cdot d(T z, T t)^{0} \\
& \cdot d\left(T z, T^{2} z\right)^{0} \cdot d\left(T t, T^{2} t\right)^{0} \\
= & d(z, t) \cdot 1 \cdot 1 \cdot 1 \cdot 1 \cdot 1=d(z, t) .
\end{aligned}
$$

Notice that this case corresponds to the Banach contractivity condition particularized to $T^{2}$ instead of $T$ :

$$
\alpha(z, t) d\left(T^{2} z, T^{2} t\right) \leq k \cdot d(z, t)
$$

which appears when $\alpha(z, t)=1$ for all $z, t \in M$. Other similar cases will be discussed in Remark 23 .

The first main theorem of this work is the following one.

Theorem 12. Let $(M, d)$ be a complete metric space. A continuous hybrid-interpolative Reich-Istrățescu-type contraction $T: M \longrightarrow M$ has at least a fixed point provided that the mapping $T$ is $\alpha$-orbital admissible and there exists $z_{0} \in$ $M$ such that $\alpha\left(z_{0}, T z_{0}\right) \geq 1$

Proof. From the hypothesis, we know that there exists $z_{0} \in M$ such that $\alpha\left(\mathrm{z}_{0}, T_{0}\right) \geq 1$. Since $T$ is $\alpha$-orbital admissible, $\alpha(T$ $\left.\mathrm{z}_{0}, T^{2} \mathrm{z}_{0}\right) \geq 1$, and by an inductive reasoning, we get that $\alpha($ $\left.T^{n} z_{0}, T^{n+1} z_{0}\right) \leq 1$ for any $n \in \mathbb{N}$. Starting from this point $z_{0}$ $\in M$, we define the sequence $\left\{z_{n}\right\}$ in $M$ as follows:

$$
z_{1}=T z_{0}, z_{2}=T z_{1}=T^{2} z_{0}, \cdots, z_{n}=T z_{n-1}=T^{n} z_{0}
$$

If there is some $n \in \mathbb{N}$ satisfying that $z_{n}=z_{n+1}$, then $z_{n}$ is a fixed point of $T$, and the proof finishes here. On the contrary case, suppose that $z_{n}$ is distinct to $z_{n+1}$ for all $n \in \mathbb{N}$. $\square$

We will divide the proof into two cases, namely, $\lambda>0$ and $\lambda=0$. In both cases, we prove that the sequence $\left\{z_{n}\right\}$ is Cauchy.
Case A. For the first case, $\lambda>0$, given any $n \in \mathbb{N}$, taking in (35) $z=z_{n}$ and $t=z_{n+1}$, we have

$$
\begin{aligned}
d\left(z_{n+2}, z_{n+3}\right) \leq & \alpha\left(z_{n+2}, z_{n+3}\right) d\left(T^{2} z_{n}, T^{2} z_{n+1}\right) \leq k \cdot \mathscr{I}^{\lambda}\left(z_{n}, z_{n+1}\right)=k \\
& \cdot\left[a_{1} d\left(z_{n}, z_{n+1}\right)^{\lambda}+a_{2} d\left(z_{n}, T z_{n}\right)^{\lambda}+a_{3} d\left(z_{n+1}, T z_{n+1}\right)^{\lambda}\right. \\
& +a_{4} d\left(T z_{n}, T z_{n+1}\right)^{\lambda}+a_{5} d\left(T z_{n}, T^{2} z_{n}\right)^{\lambda} \\
& \left.+\delta d\left(T z_{n+1}, T^{2} z_{n+1}\right)^{\lambda}\right]^{1 / \lambda}=k \cdot\left[a_{1} d\left(z_{n}, z_{n+1}\right)^{\lambda}\right. \\
& +a_{2} d\left(z_{n}, z_{n+1}\right)^{\lambda}+a_{3} d\left(z_{n+1}, z_{n+2}\right)^{\lambda}+a_{4} d\left(z_{n+1}, z_{n+2}\right)^{\lambda} \\
& \left.+a_{5} d\left(z_{n+1}, z_{n+2}\right)^{\lambda}+\delta d\left(z_{n+2}, z_{n+3}\right)^{\lambda}\right]^{1 / \lambda} \\
= & \cdot \cdot\left(a_{1}+a_{2}\right) d\left(z_{n}, z_{n+1}\right)^{\lambda}+\left(a_{3}+a_{4}+a_{5}\right) d\left(z_{n+1}, z_{n+2}\right)^{\lambda} \\
& \left.+\delta d\left(z_{n+2}, z_{n+3}\right)^{\lambda}\right]^{1 / \lambda} \leq k \\
& \cdot\left[\left(a_{1}+a_{2}+a_{3}+a_{4}+a_{5}\right) \max \left\{d\left(z_{n}, z_{n+1}\right)^{\lambda}, d\left(z_{n+1}, z_{n+2}\right)^{\lambda}\right\}\right. \\
& \left.+\delta d\left(z_{n+2}, z_{n+3}\right)^{\lambda}\right]^{1 / \lambda} .
\end{aligned}
$$

Using the power of $\lambda$,

$$
\begin{aligned}
d\left(z_{n+2}, z_{n+3}\right)^{\lambda} \leq & k^{\lambda}\left(a_{1}+a_{2}+a_{3}+a_{4}+a_{5}\right) \max \\
& \cdot\left\{d\left(z_{n}, z_{n+1}\right)^{\lambda}, d\left(z_{n+1}, z_{n+2}\right)^{\lambda}\right\} \\
& +k^{\lambda} \delta d\left(z_{n+2}, z_{n+3}\right)^{\lambda} .
\end{aligned}
$$

Therefore,

$$
\begin{aligned}
(1 & \left.-k^{\lambda} \delta\right) d\left(z_{n+2}, z_{n+3}\right)^{\lambda} \\
& \leq k^{\lambda}\left(a_{1}+a_{2}+a_{3}+a_{4}+a_{5}\right) \max \left\{d\left(z_{n}, z_{n+1}\right)^{\lambda}, d\left(z_{n+1}, z_{n+2}\right)^{\lambda}\right\} \\
& \leq k^{\lambda}(1-\delta)\left(\max \left\{d\left(z_{n}, z_{n+1}\right), d\left(z_{n+1}, z_{n+2}\right)\right\}\right)^{\lambda},
\end{aligned}
$$

which means that, for all $n \in \mathbb{N}$,

$$
d\left(z_{n+2}, z_{n+3}\right)^{\lambda} \leq \frac{k^{\lambda}(1-\delta)}{1-k^{\lambda} \delta} \cdot\left(\max \left\{d\left(z_{n}, z_{n+1}\right), d\left(z_{n+1}, z_{n+2}\right)\right\}\right)^{\lambda},
$$

or, equivalently,

$$
d\left(z_{n+2}, z_{n+3}\right) \leq\left(\frac{k^{\lambda}(1-\delta)}{1-k^{\lambda} \delta}\right)^{1 / \lambda} \cdot \max \left\{d\left(z_{n}, z_{n+1}\right), d\left(z_{n+1}, z_{n+2}\right)\right\}
$$

Let us denote

$$
c=\left(\frac{k^{\lambda}(1-\delta)}{1-k^{\lambda} \delta}\right)^{1 / \lambda}
$$


Clearly,

$$
c=\left(\frac{k^{\lambda}(1-\delta)}{1-k^{\lambda} \delta}\right)^{1 / \lambda}=\left(\frac{k^{\lambda}-k^{\lambda} \delta}{1-k^{\lambda} \delta}\right)^{1 / \lambda}<1 .
$$

This proves that there is $c \in 0,1)$ such that

$$
d\left(z_{n+2}, z_{n+3}\right) \leq c \max \left\{d\left(z_{n}, z_{n+1}\right), d\left(z_{n+1}, z_{n+2}\right)\right\} \text { for all } n \in \mathbb{N} \text {. }
$$

Lemma 5 concludes that $\left\{z_{n}\right\}_{n \in \mathbb{N}}$ is a Cauchy sequence in $(M, d)$.

Case B. For the case $\lambda=0$, given $n \in \mathbb{N}$, taking $z=z_{n}$ and $t=z_{n+1}$, we have

$$
\begin{aligned}
\mathscr{I}^{\lambda}\left(z_{n}, z_{n+1}\right)= & d\left(z_{n}, z_{n+1}\right)^{a_{1}} \cdot d\left(z_{n}, T z_{n}\right)^{a_{2}} \cdot d\left(z_{n+1}, T z_{n+1}\right)^{a_{3}} \\
& \cdot d\left(T z_{n}, T z_{n+1}\right)^{a_{4}} \cdot d\left(T z_{n}, T^{2} z_{n}\right)^{a_{5}} \\
& \cdot d\left(T z_{n+1}, T^{2} z_{n+1}\right)^{\delta}=d\left(z_{n}, z_{n+1}\right)^{a_{1}} \\
& \cdot d\left(z_{n}, z_{n+1}\right)^{a_{2}} \cdot d\left(z_{n+1}, z_{n+2}\right)^{a_{3}} \cdot d\left(z_{n+1}, z_{n+2}\right)^{a_{4}} \\
& \cdot d\left(z_{n+1}, z_{n+2}\right)^{a_{5}} \cdot d\left(z_{n+2}, z_{n+3}\right)^{\delta} \\
= & d\left(z_{n}, z_{n+1}\right)^{a_{1}+a_{2}} \cdot d\left(z_{n+1}, z_{n+2}\right)^{a_{3}+a_{4}+a_{5}} \\
& \cdot d\left(z_{n+2}, z_{n+3}\right)^{\delta} .
\end{aligned}
$$

Hence, the contractivity condition (35) means that

$$
\begin{aligned}
d\left(z_{n+2}, z_{n+3}\right) \leq & \alpha\left(z_{n+2}, z_{n+3}\right) d\left(z_{n+2}, z_{n+3}\right) \\
= & \alpha\left(z_{n+2}, z_{n+3}\right) d\left(T^{2} z_{n}, T^{2} z_{n+1}\right) \\
\leq & k \cdot \mathscr{I}^{\lambda}\left(z_{n}, z_{n+1}\right)=k \cdot d\left(z_{n}, z_{n+1}\right)^{a_{1}+a_{2}} \\
& \cdot d\left(z_{n+1}, z_{n+2}\right)^{a_{3}+a_{4}+a_{5}} \cdot d\left(z_{n+2}, z_{n+3}\right)^{\delta} .
\end{aligned}
$$

If $\delta=1$, then $a_{1}=a_{2}=a_{3}=a_{4}=a_{5}=0$, and the previous inequality would be

$$
0<d\left(z_{n+2}, z_{n+3}\right) \leq k \cdot d\left(z_{n+2}, z_{n+3}\right),
$$

which is false. Then, necessarily $\delta<1$, so $\sum_{i=1}^{5} a_{i}=1-\delta>0$, which means that the numbers

$$
\begin{gathered}
\alpha=\frac{a_{1}+a_{2}}{1-\delta}, \\
\beta=\frac{a_{3}+a_{4}+a_{5}}{1-\delta}
\end{gathered}
$$

satisfy $\alpha+\beta=1$. As a result, property (53) is equivalent to

$$
\begin{aligned}
d\left(z_{n+2}, z_{n+3}\right) \leq & k \cdot d\left(z_{n}, z_{n+1}\right)^{a_{1}+a_{2}} \cdot d\left(z_{n+1}, z_{n+2}\right)^{a_{3}+a_{4}+a_{5}} \\
& \cdot d\left(z_{n+2}, z_{n+3}\right)^{\delta} \Leftrightarrow d\left(z_{n+2}, z_{n+3}\right)^{1-\delta} \leq k \\
& \cdot d\left(z_{n}, z_{n+1}\right)^{a_{1}+a_{2}} \cdot d\left(z_{n+1}, z_{n+2}\right)^{a_{3}+a_{4}+a_{5}} \Leftrightarrow d\left(z_{n+2}, z_{n+3}\right) \\
\leq & k^{1 /(1-\delta)} \cdot d\left(z_{n}, z_{n+1}\right)^{\left(a_{1}+a_{2}\right) /(1-\delta)} \\
& \cdot d\left(z_{n+1}, z_{n+2}\right)^{\left(a_{3}+a_{4}+a_{5}\right) /(1-\delta)} \Leftrightarrow d\left(z_{n+2}, z_{n+3}\right) \\
\leq & k^{1 /(1-\delta)} \cdot d\left(z_{n}, z_{n+1}\right)^{\alpha} \cdot d\left(z_{n+1}, z_{n+2}\right)^{\beta},
\end{aligned}
$$

for all $n \in \mathbb{N}$. Taking into account that $k \in 0,1$ ),

$$
0<1-\delta \leq 1 \Rightarrow 1 \leq \frac{1}{1-\delta} \Rightarrow k^{1 /(1-\delta)} \leq k<1 .
$$

Since $0<k^{1 /(1-\delta)}<1$ and $\alpha+\beta=1$, Corollary 9 finally concludes that $\left\{z_{n}\right\}_{n \in \mathbb{N}}$ is a Cauchy sequence in $(M, d)$.

In both cases $(\lambda>0$ and $\lambda=0)$, we have demonstrated that $\left\{z_{n}\right\}_{n \in \mathbb{N}}$ is a Cauchy sequence in $(M, d)$. As it is complete, then there exists a point $u \in M$ such that $\left\{z_{n}\right\} \longrightarrow u$ as $n \longrightarrow \infty$. Moreover, due to the continuity of the mapping $T$, we conclude that $T u=u$; that is, $u$ is a fixed point of $T$.

Remark 14. Notice that in the previous proof we have shown, without using the continuity of the mapping $T$; that is, in both cases $(\lambda>0$ and $\lambda=0)$, the Picard sequence $\left\{z_{n}\right\}_{n \in \mathbb{N}}$ is Cauchy in the metric space $(M, d)$. Using its completeness, it follows that there exists a point $u \in M$ such that \{ $\left.z_{n}\right\} \longrightarrow u$ as $n \longrightarrow \infty$. Then, we can use this argument in the next results because the continuity of the mapping $T$ is only used in the last part of the proof.

When $\lambda=0$ and $\sum_{i=1}^{5} a_{i}+\delta<1$, the statement given by Theorem 12 is false. For instance, if $a_{1}=a_{2}=a_{3}=a_{4}=a_{5}=$ $\delta=0$, the contractive condition (36) does not provide any kind of control on $d\left(T^{2} z, T^{2} t\right)$ because the value of $\mathscr{I}^{0}(z, t$ ) is always 1 (all exponents are zero). However, even if all constants $a_{1}, a_{2}, a_{3}, a_{4}, a_{5}$, and $\delta$ are strictly positive, the operator $T$ could be fixed point free, as we show in the next example.

Example 15. Let $M=\{0,0.001,0.002,0.003,0.004\}$ be endowed with the Euclidean distance $d$. Let $T: M \longrightarrow M$ be defined by

$$
T z= \begin{cases}z+0.001, & \text { if } z<0.004 \\ 0, & \text { if } z=0.004\end{cases}
$$

Clearly, $d(z, t) \leq 0.004$, so

$$
d\left(T^{2} z, T^{2} t\right) \leq 0.004 \text { for all } z, t \in M .
$$

If we define

$$
k=0.95, a_{1}=a_{2}=a_{3}=a_{4}=a_{5}=\delta=0.05,
$$


the values of $k \cdot \mathscr{I}^{0}(z, t)$ are given in the following table:

\begin{tabular}{c|ccccc}
$\mathrm{k} \cdot \mathscr{I}^{0}(\mathrm{z}, \mathrm{t})$ & 0 & 0.001 & 0.002 & 0.003 & 0.004 \\
\hline 0 & 0 & 0.119598 & 0.128182 & 0.143067 & 0.137382 \\
0.001 & 0.119598 & 0 & 0.119598 & 0.137382 & 0.140196 \\
0.002 & 0.128182 & 0.119598 & 0 & 0.128182 & 0.140196 \\
0.003 & 0.143067 & 0.137382 & 0.128182 & 0 & 0.147242 \\
0.004 & 0.137382 & 0.140196 & 0.140196 & 0.147242 & 0
\end{tabular}

As a consequence, if $\alpha$ is given by $\alpha(z, t)=1$ for all $z, t$ $\in M$, then the mapping $T$ satisfies

$$
d\left(T^{2} z, T^{2} t\right) \leq 0.004<0.1<k \cdot \mathscr{I}^{0}(z, t)
$$

for all distinct $z, t \in M$. Therefore, $T$ is a hybrid-interpolative Reich-Istrățescu-type contraction. Furthermore, $(M, d)$ is complete, $T$ is $\alpha$-orbital admissible, and there exists $z_{0} \in M$ such that $\alpha\left(z_{0}, T z_{0}\right) \geq 1$. However, $T$ is fixed point free.

Remark 16. The reason why Theorem 12 could fail when $\lambda$ $=0$ and $\sum_{i=1}^{5} a_{i}+\delta<1$ is the following one: a sequence $\left\{z_{n}\right\}_{n \in \mathbb{N}}$ satisfying

$$
d\left(z_{n+2}, z_{n+3}\right) \leq k \cdot d\left(z_{n}, z_{n+1}\right)^{\alpha} \cdot d\left(z_{n+1}, z_{n+2}\right)^{\beta} \text { for all } n \in \mathbb{N}
$$

could be non-Cauchy when $\alpha+\beta<1$. For instance, let $\left\{z_{n}\right\}_{n \in \mathbb{N}}$ be the sequence defined by

$$
z_{n}=0.25 n \text { for all } n \in \mathbb{N} \text {. }
$$

If $M=\left\{z_{n}: n \in \mathbb{N}\right\} \subset[0, \infty)$ is endowed with the Euclidean distance, then

$d\left(z_{n}, z_{n+1}\right)=\left|z_{n+1}-z_{n}\right|=|0.25(n+1)-0.25 n|=0.25$ for all $n \in \mathbb{N}$.

Therefore, if we take

$$
\begin{gathered}
k=\delta=\frac{1}{2}, \\
a_{1}=a_{2}=\frac{1}{16}, \\
a_{3}=a_{4}=a_{5}=\frac{1}{24},
\end{gathered}
$$

then, for all $n \in \mathbb{N}$,

$$
\begin{gathered}
k \cdot d\left(z_{n}, z_{n+1}\right)^{\left(a_{1}+a_{2}\right) /(1-\delta)} \cdot d\left(z_{n+1}, z_{n+2}\right)^{\left(a_{3}+a_{4}+a_{5}\right) /(1-\delta)} \\
\quad=0.5 \cdot 0.25^{0.25} \cdot 0.25^{0.25}=0.25=d\left(z_{n+2}, z_{n+3}\right) .
\end{gathered}
$$

However, the sequence $\left\{z_{n}\right\}_{n \in \mathbb{N}}$ positively diverges, so it is not Cauchy.

If the operator $T$ is continuous on $M$, then the composition $T^{2}=T \circ T$ also is. However, the mapping $T^{2}$ could be continuous even if $T$ is not continuous. In this case, we can replace the continuity of the mapping $T$ with a weaker condition, namely, the continuity of $T^{2}$, whose set of fixed points is nonempty, as it is shown in the next statement.

Theorem 17. Let $(M, d)$ be a complete metric space and $T$ $: M \longrightarrow M$ be a hybrid-interpolative Reich-Istrățescu-type contraction such that $T^{2}$ is continuous. If, in addition,

(1) $T$ is $\alpha$-orbital admissible

(2) there exists $z_{0} \in M$ such that $\alpha\left(z_{0}, T z_{0}\right) \geq 1$

(3) $\alpha(u, T u) \geq 1$ for $u \in *$ Fix $_{T^{2}}(M)$

then $T$ has a fixed point.

Proof. Let $\left\{z_{n}=T^{n} z_{0}\right\}_{n \in \mathbb{N}}$ be the Picard sequence of $T$ whose initial point is $z_{0}$. In Remark 14, we commented that this sequence has a limit $u \in M$. Since the mapping $T^{2}$ is continuous, then

$$
d\left(u, T^{2} u\right)=\lim _{n \longrightarrow \infty} d\left(z_{n+2}, T^{2} u\right)=\lim _{n \longrightarrow \infty} d\left(T^{2} z_{n}, T^{2} u\right)=0
$$

Thereby, $T^{2} u=u$; that is, $u$ is a fixed point of $T^{2}$. As a result, the mapping $T^{2}$ has at least one fixed point; that is, the set $* \operatorname{Fix}_{T^{2}}(M)$ is nonempty. Furthermore, $T^{3} u=T u$. In order to check that $u$ is also a fixed point of $T$, suppose, by contradiction, that $u \neq T u$. In this case, $T u$ is not a fixed point either because, in such a case, $T u=T^{2} u=u$, which is false. Then, $u, T u \in M \backslash * \operatorname{Fix}_{T}(M)$.

Case A. If $\lambda>0$, then

$$
\begin{aligned}
\mathscr{I}^{\lambda}(u, T u)= & {\left[a_{1} d(u, T u)^{\lambda}+a_{2} d(u, T u)^{\lambda}+a_{3} d\left(T u, T^{2} u\right)^{\lambda}\right.} \\
& \left.+a_{4} d\left(T u, T^{2} u\right)^{\lambda}+a_{5} d\left(T u, T^{2} u\right)^{\lambda}+\delta d\left(T^{2} u, T^{3} u\right)^{\lambda}\right]^{1 / \lambda} \\
= & {\left[a_{1} d(u, T u)^{\lambda}+a_{2} d(u, T u)^{\lambda}+a_{3} d(T u, u)^{\lambda}+a_{4} d(T u, u)^{\lambda}\right.} \\
& \left.+a_{5} d(T u, u)^{\lambda}+\delta d(u, T u)^{\lambda}\right]^{1 / \lambda} \\
= & {\left[\left(a_{1}+a_{2}+a_{3}+a_{4}+a_{5}+\delta\right) d(u, T u)^{\lambda}\right]^{1 / \lambda} } \\
\leq & {\left[d(u, T u)^{\lambda}\right]^{1 / \lambda}=d(u, T u) . }
\end{aligned}
$$

Since $\alpha(u, T u) \geq 1$, then from (35),

$$
\begin{aligned}
0 & <d(u, T u) \leq \alpha(u, T u) d(u, T u)=\alpha(u, T u) d\left(T^{2} u, T^{3} u\right) \\
& \leq k \cdot \mathscr{I}^{\lambda}(u, T u) \leq k \cdot d(u, T u),
\end{aligned}
$$

which is a contradiction. Then, $T u=u$, so $u$ is a fixed point of $T$. 
Case B. If $\lambda=0$, then

$$
\begin{aligned}
\mathscr{I}^{0}(u, T u)= & d(u, T u)^{a_{1}} \cdot d(u, T u)^{a_{2}} \cdot d\left(T u, T^{2} u\right)^{a_{3}} \\
& \cdot d\left(T u, T^{2} u\right)^{a_{4}} \cdot d\left(T u, T^{2} u\right)^{a_{5}} \cdot d\left(T^{2} u, T^{3} u\right)^{\delta} \\
= & d(u, T u)^{a_{1}} \cdot d(u, T u)^{a_{2}} \cdot d(T u, u)^{a_{3}} \cdot d(T u, u)^{a_{4}} \\
& \cdot d(T u, u)^{a_{5}} \cdot d(u, T u)^{\delta}=d(u, T u)^{a_{1}+a_{2}+a_{3}+a_{4}+a_{5}+\delta} \\
= & d(u, T u),
\end{aligned}
$$

and the argument of the previous case can be repeated here.

For the case $\lambda>0$, by adding a supplementary condition, we can show the uniqueness of the fixed point.

Theorem 18. Under the hypotheses of Theorem 17, if we suppose that the contractivity condition (35) holds for all distinct points $u, v \in M$ and also that $\alpha(u, v) \geq 1$ for every $u, v \in * F i$ $x_{T}(M)$, then $T$ has a unique fixed point.

Proof. Theorem 17 guarantees that the set of fixed points of $T$ is nonempty. Suppose that $T$ has two distinct fixed points $u, v \in * \operatorname{Fix}_{T}(M)$

Case A. If $\lambda>0$, replacing such points in (35), we obtain

$$
\begin{aligned}
\mathscr{I}^{\lambda}(u, v)= & {\left[a_{1} d(u, v)^{\lambda}+a_{2} d(u, T u)^{\lambda}+a_{3} d(v, T v)^{\lambda}\right.} \\
& \left.+a_{4} d(T u, T v)^{\lambda}+a_{5} d\left(T u, T^{2} u\right)^{\lambda}+\delta d\left(T v, T^{2} v\right)^{\lambda}\right]^{1 / \lambda} \\
= & {\left[a_{1} d(u, v)^{\lambda}+a_{4} d(u, v)^{\lambda}\right]^{1 / \lambda}=\left(a_{1}+a_{4}\right)^{1 / \lambda} d(u, v) } \\
\leq & d(u, v) .
\end{aligned}
$$

Therefore,

$$
\begin{aligned}
d(u, v) & \leq \alpha(u, v) d(u, v)=\alpha(u, v) d\left(T^{2} u, T^{2} v\right) \\
& \leq k \cdot \mathscr{I}^{\lambda}(u, v) \leq k \cdot d(u, v)<d(u, v),
\end{aligned}
$$

which is impossible. Then, $T$ cannot have two distinct fixed points.

Case B. If $\lambda=0$, then

$$
\begin{aligned}
\mathscr{I}^{0}(u, v)= & d(u, v)^{a_{1}} \cdot d(u, T u)^{a_{2}} \cdot d(v, T v)^{a_{3}} \cdot d(T u, T v)^{a_{4}} \\
& \cdot d\left(T u, T^{2} u\right)^{a_{5}} \cdot d\left(T v, T^{2} v\right)^{\delta}=d(u, v)^{a_{1}} \cdot 0^{a_{2}} \\
& \cdot 0^{a_{3}} \cdot d(u, v)^{a_{4}} \cdot 0^{a_{5}} \cdot 0^{\delta} .
\end{aligned}
$$

If $a_{2}>0$ or $a_{3}>0$ or $a_{5}>0$ or $\delta>0$, then $\mathscr{I}^{0}(u, v)=0$, so the contractivity condition (35) leads to

$$
d(u, v) \leq \alpha(u, v) d(u, v)=\alpha(u, v) d\left(T^{2} u, T^{2} v\right) \leq k \cdot \mathscr{I}^{\lambda}(u, v)=0,
$$

which is false because $u$ and $v$ are distinct points. On the contrary, if $a_{2}=a_{3}=a_{5}=\delta=0$, we agreed that $0^{a_{2}}=0^{a_{3}}=$ $0^{a_{5}}=0^{\delta}=1$, so

$$
\begin{gathered}
a_{1}+a_{4}=\sum_{i=1}^{5} a_{i}+\delta=1, \\
\mathscr{I}^{0}(u, v)=d(u, v)^{a_{1}+a_{4}}=d(u, v) .
\end{gathered}
$$

The argument shown in (73) proves that this case is also impossible, so the mapping $T$ cannot have two distinct fixed points in any case.

Also, a particular result holds for the case $\lambda=0$; more exactly, we can remove the continuity conditions of $T$ or $T^{2}$.

Theorem 19. Let $(M, d)$ be a complete metric space, and let $T: M \longrightarrow M$ be a hybrid-interpolative Reich-Istrățescu-type contraction for $\lambda=0$ such that $a_{1}>0$ or $a_{2}>0$ or $a_{5}>0$. If we suppose that

(i) $T$ is $\alpha$-orbital admissible

(ii) there exists $z_{0} \in M$ such that $\alpha\left(z_{0}, T z_{0}\right) \geq 1$

(iii) for any sequence $\left\{z_{n}\right\}_{n \in \mathbb{N}}$ in $M$ such that $\alpha\left(z_{n}, z_{n+1}\right.$ )$\geq 1$ for $n \in \mathbb{N}$ and $\left\{z_{n}\right\} \longrightarrow$ as $n \longrightarrow \infty$, we have that $\alpha\left(z_{n}, u\right) \geq 1$ for all $n \in \mathbb{N}$

then $T$ has a fixed point.

Proof. Following the proof of Theorem 12, we considered the Picard sequence $z_{n}=T^{n} z_{0}$ for all $n \in \mathbb{N}$. If this sequence contains a fixed point, the proof is finished. On the contrary case, we have shown that it is a Cauchy sequence on $(M, d$ ), so it converges to a point $u \in M$. To prove that $u$ is a fixed point of $T$, suppose, by contradiction, that $u \neq T u$.

Without loss of generality, we can assume that $\left\{z_{n}\right\}_{n \in \mathbb{N}}$ satisfies $z_{n} \neq z_{m}$ for all $n, m \in \mathbb{N}$ such that $n \neq m$. In this case, there is $n_{0} \in \mathbb{N}$ such that $z_{n}$ and $u$ are distinct and they are not fixed points of $T$ for all $n \geq n_{0}$. Let us check that $u$ is a fixed point of $T^{2}$. Indeed, for all $n \geq n_{0}$,

$$
\begin{aligned}
d\left(z_{n+2}, T^{2} u\right) \leq & \alpha\left(z_{n}, u\right) d\left(T^{2} z_{n}, T^{2} u\right) \leq k \cdot \mathscr{I}^{0}\left(z_{n}, u\right) \\
= & k \cdot d\left(z_{n}, u\right)^{a_{1}} \cdot d\left(z_{n}, T z_{n}\right)^{a_{2}} \cdot d(u, T u)^{a_{3}} \\
& \cdot d\left(T z_{n}, T u\right)^{a_{4}} \cdot d\left(T z_{n}, T^{2} z_{n}\right)^{a_{5}} \\
& \cdot d\left(T u, T^{2} u\right)^{\delta}=k \cdot d\left(z_{n}, u\right)^{a_{1}} \cdot d\left(z_{n}, z_{n+1}\right)^{a_{2}} \\
& \cdot d(u, T u)^{a_{3}} \cdot d\left(z_{n+1}, T u\right)^{a_{4}} \cdot d\left(z_{n+1}, z_{n+2}\right)^{a_{5}} \\
& \cdot d\left(T u, T^{2} u\right)^{\delta} .
\end{aligned}
$$

Since $a_{1}>0$ or $a_{2}>0$ or $a_{5}>0$, letting $n \longrightarrow \infty$ in (77), we find out that $d\left(u, T^{2} u\right)=0$; that is, $u$ is a fixed point of $T^{2}$. Now, following the lines from Theorem 17, we obtain a contradiction that proves that $u$ is also a fixed point of $T$. $\square$ 
Let us show an example in which we can deduce the existence of a fixed point from Theorem 19 even when $T$ and $T^{2}$ are not continuous at the same time.

Example 20. Let $(M, d)$ be the complete metric space where $M=[-1,1]$, and $d: M \times M \longrightarrow 0, \infty)$ is the metric $d(z, t)$ $=|z-t|$ for all $z, t \in M$. Let $T: M \longrightarrow M$ be the mapping defined for each $z \in M$ by

$$
T z= \begin{cases}\sqrt{1-z^{2}}, & \text { if } z \in[-1,0), \\ \frac{z^{2}}{2}, & \text { if } z \in[0,1] .\end{cases}
$$

Its self-composition is given by

$$
T^{2} z= \begin{cases}\frac{1-z^{2}}{2}, & \text { if } z \in[-1,0), \\ \frac{z^{4}}{8}, & \text { if } z \in[0,1] .\end{cases}
$$

Clearly, $T$ and $T^{2}$ are not continuous mappings. Next, let us show that $T$ is a hybrid-interpolative Reich-Istrățescutype contraction w.r.t. the function $\alpha: M \times M \longrightarrow[0, \infty)$ defined as follows:

$$
\alpha(z, t)= \begin{cases}\frac{3}{2}, & \text { if } z, t \in[0,1] \\ 1, & \text { if } z=-1, t=1 \\ 0, & \text { otherwise. }\end{cases}
$$

Let us take

$$
k=\frac{3}{4}, a_{1}=a_{4}=\frac{1}{2}, a_{2}=a_{3}=a_{5}=\delta=0 .
$$

Assumptions (i), (ii), and (iii) of Theorem 19 are satisfied. Furthermore, the contractivity condition can be checked as follows:

(a) For every $z, t \in 0,1]$, we have

$$
\begin{aligned}
\alpha(z, t) d\left(T^{2} z, T^{2} t\right) & =\frac{3}{2}\left|\frac{z^{4}}{8}-\frac{t^{4}}{8}\right|=\frac{3\left|z^{2}-t^{2}\right|\left(z^{2}+t^{2}\right)}{16} \\
& \leq \frac{3}{8}\left|z^{2}-t^{2}\right|=\frac{3}{8} \cdot \sqrt{\left|z^{2}-t^{2}\right|} \cdot \sqrt{\left|z^{2}-t^{2}\right|} \\
& =\frac{3}{8} \cdot \sqrt{|z+t| \cdot|z-t|} \cdot \sqrt{2 \cdot \frac{\left|z^{2}-t^{2}\right|}{2}} \\
& =\frac{3}{8} \cdot \sqrt{2} \cdot \sqrt{|z+t|} \cdot \sqrt{|z-t|} \cdot \sqrt{\left|\frac{z^{2}}{2}-\frac{t^{2}}{2}\right|} \\
& \leq \frac{3}{8} \cdot \sqrt{2} \cdot \sqrt{2} \cdot|z-t|^{1 / 2} \cdot\left|\frac{z^{2}}{2}-\frac{t^{2}}{2}\right|^{1 / 2} \\
& =\frac{3}{4} \cdot d(z, t)^{1 / 2} \cdot d(T z, T t)^{1 / 2},
\end{aligned}
$$

(b) For $z=-1$ and $t=1$, we have

$$
\begin{aligned}
& \alpha(-1,1) d\left(T^{2}(-1), T^{2} 1\right)=\frac{1}{8}<\frac{3}{4} \\
& \quad=\frac{3}{4} \cdot d(-1,1)^{1 / 2} \cdot d(T(-1), T 1)^{1 / 2} .
\end{aligned}
$$

(c) For all other cases,

$$
\alpha(z, t) d\left(T^{2} z, T^{2} t\right)=0 \leq \frac{3}{4} \cdot d(z, t)^{1 / 2} \cdot d(T z, T t)^{1 / 2} .
$$
point.

Hence, from Theorem 19, we conclude that $T$ has a fixed

\section{Consequences}

In the field of fixed point theory, it is commonly accepted that a contractivity condition is all the more general if it has more possibilities of being particularized, giving rise to versions of already known theorems. Therefore, in order to show the power of the main introduced results, in this section we are going to illustrate several contexts in which they can be applied.

The first important framework appears when the mapping $\alpha: M \times M \longrightarrow[0, \infty)$ constantly takes the value 1 ; that is, $\alpha(z, t)=1$ for each $z, t \in M$. In this case, the hypotheses about the $\alpha$-orbital admissibility and the existence of a point $z_{0} \in M$ such that $\alpha\left(z_{0}, T z_{0}\right) \geq 1$ are trivial.

Corollary 21. Let $(M, d)$ be a complete metric space, and let $T: M \longrightarrow M$ be a mapping such that

(i) either $T$ or $T^{2}$ is continuous

(ii) for some $\lambda \in 0, \infty)$, there exist a constant $k \in 0,1)$ and six numbers $a_{1}, a_{2}, a_{3}, a_{4}, a_{5}, \delta \in[0,1]$ such that, for all distinct $z, t \in M \backslash * \operatorname{Fix}_{T}(M)$,

$$
d\left(T^{2} z, T^{2} t\right) \leq k \cdot \mathscr{I}^{\lambda}(z, t)
$$

where $\mathscr{I}^{\lambda}(z, t)$ is defined by (36) and

$$
\begin{aligned}
& \sum_{i=1}^{5} a_{i}+\delta \leq 1, \quad \text { if } \lambda>0 \\
& \sum_{i=1}^{5} a_{i}+\delta=1, \quad \text { if } \lambda=0 .
\end{aligned}
$$

Then, $T$ has a fixed point. 
In addition to this, for the case $\lambda>0$, if we suppose that the contractivity condition (85) holds for all distinct points $u, v \in M$, then $T$ has a unique fixed point.

Proof. It follows from Theorems 12, 17, and 18 applied to the case in which $\alpha(z, t)=1$ for each $z, t \in M . \square$

The following result follows by choosing in the set of constants $\left\{a_{1}, a_{2}, a_{3}, a_{4}, a_{5}, \delta\right\}$ one of them as 1 and the other ones as 0 . Notice that six corollaries are being summarized into only one.

Corollary 22. Let $(M, d)$ be a complete metric space, let $\alpha$ $: M \times M \longrightarrow[0, \infty)$ be a function, and let $T: M \longrightarrow M$ be a mapping such that

(i) either $T$ or $T^{2}$ is continuous, $T$ is $\alpha$-orbital admissible, and there exists $z_{0} \in M$ such that $\alpha\left(z_{0}, T z_{0}\right) \geq 1$

(ii) there exists a constant $k \in 0,1)$ such that at least one of the following conditions is fulfilled for all distinct $z, t \in M \backslash * \operatorname{Fix}_{T}(M)$ :

$$
\begin{gathered}
\alpha(z, t) d\left(T^{2} z, T^{2} t\right) \leq k \cdot d(z, t), \\
\alpha(z, t) d\left(T^{2} z, T^{2} t\right) \leq k \cdot d(z, T z), \\
\alpha(z, t) d\left(T^{2} z, T^{2} t\right) \leq k \cdot d(t, T t), \\
\alpha(z, t) d\left(T^{2} z, T^{2} t\right) \leq k \cdot d(T z, T t), \\
\alpha(z, t) d\left(T^{2} z, T^{2} t\right) \leq k \cdot d\left(T z, T^{2} z\right), \\
\alpha(z, t) d\left(T^{2} z, T^{2} t\right) \leq k \cdot d\left(T t, T^{2} t\right) .
\end{gathered}
$$

Then, $T$ has a fixed point.

Proof. This result corresponds to the case $\lambda>0$ in Theorem 12 ( $T$ continuous) or Theorem 17 ( $T^{2}$ continuous) when the contractivity condition (35) is considered by using the following respective choices for constants:

$$
\begin{aligned}
& a_{1}=1, a_{2}=a_{3}=a_{4}=a_{5}=\delta=0 ; \\
& a_{2}=1, a_{1}=a_{3}=a_{4}=a_{5}=\delta=0, \\
& a_{3}=1, a_{1}=a_{2}=a_{4}=a_{5}=\delta=0, \\
& a_{4}=1, a_{1}=a_{2}=a_{3}=a_{5}=\delta=0, \\
& a_{5}=1, a_{1}=a_{2}=a_{3}=a_{4}=\delta=0, \\
& \delta=1, a_{1}=a_{2}=a_{3}=a_{4}=a_{5}=0 .
\end{aligned}
$$

Remark 23. Notice that we would have obtained the same six contractivity conditions (87) for the case $\lambda=0$ under the six cases given by (88).
The following result follows by using $\alpha(z, t)=1$ for each $z, t \in M$ in the previous statement.

Corollary 24. Let $(M, d)$ be a complete metric space, and let $T: M \longrightarrow M$ be a mapping such that

(i) either $T$ or $T^{2}$ is continuous

(ii) there exists a constant $k \in 0,1)$ such that at least one of the following conditions is fulfilled for all distinct $z, t \in M \backslash * \operatorname{Fix}_{T}(M)$ :

$$
\begin{gathered}
d\left(T^{2} z, T^{2} t\right) \leq k \cdot d(z, t), \\
d\left(T^{2} z, T^{2} t\right) \leq k \cdot d(z, T z), \\
d\left(T^{2} z, T^{2} t\right) \leq k \cdot d(t, T t), \\
d\left(T^{2} z, T^{2} t\right) \leq k \cdot d(T z, T t), \\
d\left(T^{2} z, T^{2} t\right) \leq k \cdot d\left(T z, T^{2} z\right), \\
d\left(T^{2} z, T^{2} t\right) \leq k \cdot d\left(T t, T^{2} t\right) .
\end{gathered}
$$

Then, T has a fixed point.

Particular cases are especially interesting, like in the following case, in which it is not necessary to assume the continuity of the mapping $T$.

Corollary 25 (Istrățescu $[18,19])$. Let $(M, d)$ be a complete metric space, and let $T: M \longrightarrow M$ be a continuous mapping such that there exist $a, b \in(0,1)$ with $a+b<1$ satisfying

$$
d\left(T^{2} z, T^{2} t\right) \leq a \cdot d(z, t)+b \cdot d(T z, T t) \text { for all } z, t \in M \text {. }
$$

Then, T has a unique fixed point.

Proof. Let us consider the choices $\lambda=1$ and $\alpha(z, t)=1$ for each $z, t \in M$. Let $k=a+b \in(0,1)$, and let

$$
a_{1}=\frac{a}{k}, a_{4}=\frac{b}{k}, a_{2}=a_{3}=a_{5}=\delta=0 .
$$

Then, for each distinct $z, t \in M$,

$$
\begin{aligned}
k \cdot \mathscr{I}^{1}(z, t) & =k \cdot\left[\frac{a}{k} d(z, t)+\frac{b}{k} d(T z, T t)\right] \\
& =a \cdot d(z, t)+b \cdot d(T z, T t),
\end{aligned}
$$

which means that the contractivity condition

$$
\alpha(z, t) d\left(T^{2} z, T^{2} t\right) \leq k \cdot \mathscr{I}^{1}(z, t) \text { for all distinct } z, t \in M \backslash * \operatorname{Fix}_{T}(M)
$$

holds because of (90). Under this framework, the proof of Theorem 12 (using any initial point $z_{0} \in M$ ) guarantees that the Picard sequence $\left\{z_{n}=T^{n} z_{0}\right\}_{n \in \mathbb{N}}$ converges to a point 
$u_{0} \in M$. Since $T$ is continuous, then $\left\{z_{n+1}=T z_{n}\right\}_{n \in \mathbb{N}}$ converges to $T u_{0}$, so $T u_{0}=u_{0}$.

Furthermore, $T$ has a unique fixed point because if $u_{0}$ and $v_{0}$ were two distinct fixed points of $T$, then

$$
\begin{aligned}
d\left(u_{0}, v_{0}\right) & =d\left(T^{2} u_{0}, T^{2} v_{0}\right) \leq a \cdot d\left(u_{0}, v_{0}\right)+b \cdot d\left(T u_{0}, T v_{0}\right) \\
& =(a+b) d\left(u_{0}, v_{0}\right)<d\left(u_{0}, v_{0}\right),
\end{aligned}
$$

which is impossible.

In the following result, we employ a binary relation for controlling the pairs of points that must satisfy the contractivity condition. Let $\mathscr{R}$ be a binary relation on the set $M$. A mapping $T: M \longrightarrow M$ is $\mathscr{R}$-orbital admissible if $T z \mathscr{R} T t$ for all $z, t \in M$ such that $z \mathscr{R} t$. $\square$

Corollary 26. Let $(M, d)$ be a complete metric space endowed with a binary relation $\mathscr{R}$, and let $T: M \longrightarrow M$ be a continuous mapping. Assume that for some $\lambda \in 0, \infty)$, there exist a constant $k \in 0,1)$ and six numbers $a_{1}, a_{2}, a_{3}, a_{4}, a_{5}, \delta \geq 0$ satisfying (37) such that, for all distinct $z, t \in M \backslash * \operatorname{Fix}_{T}(M)$ such that $z \mathscr{R}$,

$$
d\left(T^{2} z, T^{2} t\right) \leq k \cdot \mathscr{I}^{\lambda}(z, t) .
$$

If $T$ is $\mathscr{R}$-orbital admissible and there is $z_{0} \in M$ such that $z_{0} \mathscr{R T} z_{0}$, then $T$ has at least one fixed point.

Proof. Let us consider the function $\alpha_{\mathscr{R}}: M \times M \longrightarrow[0, \infty)$ defined by

$$
\alpha_{\mathscr{R}}(z, t)= \begin{cases}1, & \text { if } z \mathscr{R} t \\ 0, & \text { otherwise }\end{cases}
$$

Then, $T$ is $\alpha_{\mathscr{R}}$-orbital admissible and there is $z_{0} \in M$ such that $\alpha_{\mathscr{R}}\left(z_{0}, T z_{0}\right)=1$. The contractivity condition (95) is equivalent to (35) under the assumptions (37). Hence, Theorem 12 is applicable

In the previous corollary, when $M$ is endowed with a binary relation $\mathscr{R}$, we can replace the completeness of the metric space by the weaker version: the metric space $(M, d$ ) is $\mathscr{R}$-increasingly complete if each $d$-Cauchy sequence $\left\{z_{n}\right\}_{n \in \mathbb{N}} \subseteq M$ satisfying that $\mathrm{z}_{n} \mathscr{R} \mathrm{z}_{n+1}$ for all $n \in \mathbb{N}$ is $d$-convergent to a point of $M$. In this case, Corollary 26 can be stated as follows.

Corollary 27. Let $(M, d)$ be a $\mathscr{R}$-increasingly complete metric space w.r.t. a binary relation $\mathscr{R}$ on $M$, and let $T: M \longrightarrow M$ be a continuous mapping. Assume that for some $\lambda \in 0, \infty)$, there exist a constant $k \in 0,1)$ and six numbers $a_{1}, a_{2}, a_{3}, a_{4}$ $, a_{5}, \delta \geq 0$ satisfying (37) such that, for all distinct $z, t \in M \backslash$
*Fix $(M)$ such that $z \mathscr{R}$,

$$
d\left(T^{2} z, T^{2} t\right) \leq k \cdot \mathscr{I}^{\lambda}(z, t) .
$$

If $T$ is $\mathscr{R}$-orbital admissible and there is $z_{0} \in M$ such that $z_{0} \mathscr{R} T z_{0}$, then $T$ has at least one fixed point.

Proof. Using the function $\alpha_{\mathscr{R}}$ defined in (96) and following the lines of the proof of Theorem 12, we get that the Picard sequence $\left\{z_{n}=T^{n} z_{0}\right\}_{n \in \mathbb{N}}$ is a Cauchy sequence in $(M, d)$ such that $\mathrm{z}_{n} \mathscr{R} T \mathrm{z}_{n}$ for all $n \in \mathbb{N}$, that is, $z_{n} \mathscr{R} z_{n+1}$ for all $n \in \mathbb{N}$. Since $(M, d)$ is an $\mathscr{R}$-increasingly complete metric space, such sequence is convergent to a point $u_{0}$ $\in M$. Then, the rest of the proof of Theorem 12 can be repeated.

The following consequence corresponds to the choice $\alpha$ $(z, t)=1$ for each $z, t \in M$ in Theorem 19. Notice that here the operator $T$ has not been continuous.

Corollary 28. Let $(M, d)$ be a complete metric space, and let $T: M \longrightarrow M$ be a mapping for which there exist a constant $k \in 0,1)$ and six numbers $a_{1}, a_{2}, a_{3}, a_{4}, a_{5}, \delta \geq 0$ such that, for all distinct $z, t \in M \backslash * \operatorname{Fix}_{T}(M)$,

$$
\begin{aligned}
d\left(T^{2} z, T^{2} t\right) \leq & k \cdot d(z, t)^{a_{1}} \cdot d(z, T z)^{a_{2}} \cdot d(t, T t)^{a_{3}} \\
& \cdot d(T z, T t)^{a_{4}} \cdot d\left(T z, T^{2} z\right)^{a_{5}} \cdot d\left(T t, T^{2} t\right)^{\delta} .
\end{aligned}
$$

Suppose that $\sum_{i=1}^{5} a_{i}+\delta=1$ and $a_{1}+a_{2}+a_{5}>0$. Then, $T$ has at least one fixed point.

Furthermore, if the contractivity condition (98) holds for all distinct points $u, v \in M$ and also $\alpha(u, v) \geq 1$ for every $u$, $v \in * \operatorname{Fix}_{T}(M)$, then $T$ has a unique fixed point.

Proof. This result follows from the case $\lambda=0$ in Theorem 19 by using the function $\alpha(z, t)=1$ for each $z, t \in M$. Notice that the condition $a_{1}+a_{2}+a_{5}>0$ means that at least one of the constants $a_{1}, a_{2}$, or $a_{5}$ is strictly positive.

To prove the uniqueness, suppose that $u, v \in * \operatorname{Fix}_{T}(M)$ are two distinct fixed points of $T$. Then,

$$
\begin{aligned}
\mathscr{F}^{0}(u, v)= & d(u, v)^{a_{1}} \cdot d(u, T u)^{a_{2}} \cdot d(v, T v)^{a_{3}} \cdot d(T u, T v)^{a_{4}} \\
& \cdot d\left(T u, T^{2} u\right)^{a_{5}} \cdot d\left(T v, T^{2} v\right)^{\delta}=d(u, v)^{a_{1}} \cdot 0^{a_{2}} \cdot 0^{a_{3}} \\
& \cdot d(u, v)^{a_{4}} \cdot 0^{a_{5}} \cdot 0^{\delta} .
\end{aligned}
$$

If $a_{2}>0$ or $a_{3}>0$ or $a_{5}>0$ or $\delta>0$, we can deduce that $\mathscr{I}^{0}(u, v)=0$, so the contractivity condition (98) leads to

$$
d(u, v)=d\left(T^{2} u, T^{2} v\right) \leq k \cdot \mathscr{I}^{\lambda}(u, v)=0,
$$


which is false because $u$ and $v$ are distinct points. On the contrary, if $a_{2}=a_{3}=a_{5}=\delta=0$, we agreed that $0^{a_{2}}=0^{a_{3}}=$ $0^{a_{5}}=0^{\delta}=1$, so

$$
\begin{gathered}
a_{1}+a_{4}=\sum_{i=1}^{5} a_{i}+\delta=1, \\
\mathscr{I}^{0}(u, v)=d(u, v)^{a_{1}+a_{4}}=d(u, v) .
\end{gathered}
$$

Therefore,

$$
d(u, v)=d\left(T^{2} u, T^{2} v\right) \leq k \cdot \mathscr{I}^{0}(u, v)=k \cdot d(u, v)<d(u, v),
$$

which is impossible. Then, $T$ cannot have two distinct fixed points.

\section{An Application to Integral Equations}

Let $C\left(\left[\tau_{0}, \Theta\right],[0,1]\right)$ be the space of all continuous functions with value on $[0,1]$ where $\Theta>0$ and for all $z, t \in C\left(\left[\tau_{0}, \Theta\right]\right.$, $[0,1])$ :

$$
d(z, t)=\sup _{\left.\tau \in \tau_{0}, \Theta\right]}|z(\tau)-t(\tau)|
$$

Obviously, the pair $(M, d)$, where $M=C\left(\left[\tau_{0}, \Theta\right],[0,1]\right)$, forms a complete metric space.

A family $\{C(\tau): \tau \in \mathbb{R}\}$ of bounded linear operators is said to be a strongly continuous cosine family if and only if

(1) $C(0)=I$ (here $I$ is the identity operator)

(2) for each $z \in M, C(\tau) z$ is strongly continuous in $\tau$ on $\mathbb{R}$

(3) $C(\tau+v)+C(\tau-v)=2 C(\tau) C(v)$ for all $\tau, v \in \mathbb{R}$

For a strongly continuous cosine family $\{C(\tau): \tau \in \mathbb{R}\}$ in $M$, we consider the following:

(1) The associated sine family $\{S(\tau): \tau \in \mathbb{R}\}$ where $S(\tau)$ $\mathrm{z}=\int_{0}^{\tau} \mathrm{C}(v) \mathrm{z} d v$, for $\tau \in \mathbb{R}$ and $z \in M$

(2) The infinitesimal generator $A: M \longrightarrow M$ defined by $A z=\left.\left(d^{2} / d \tau^{2}\right) C(\tau) z\right|_{\tau=0}$, for $z \in \mathscr{D}(A)$, where $\mathscr{D}(A)$ $=\left\{z \in M \mid C(\cdot) z \in C^{2}(\mathbb{R}, M)\right\}$

Let also $M \geq 1$ and $N>0$ be two constants such that $\mid C$ $(t) \mid \leq M$ and $|S(t)| \leq N$ for any $\tau \in\left[\tau_{0}, \Theta\right]$.
We consider the equation

$$
z^{\prime \prime}(\tau)=A z(\tau)+\xi\left(\tau, z(\tau), \int_{\tau_{0}}^{\tau} \zeta(\tau, v, z(v)) d v\right), \quad 0<\tau_{0}<\Theta
$$

$$
\left.z\left(\tau_{0}\right)=z_{0}, z^{\prime}\left(\tau_{0}\right)=w_{0}, z_{0}, w_{0} \in 0,1\right]
$$

where $\left.\xi:\left[\tau_{0}, \Theta\right] \times 0,1\right] \times \mathbb{R} \longrightarrow \mathbb{R}$ and $\left.\zeta:\left[\tau_{0}, \Theta\right] \times \tau_{0}, \Theta\right] \times$ $0,1] \longrightarrow \mathbb{R}$ are continuous functions.

A function $z \in M$ defined by

$$
\begin{aligned}
z(\tau)= & C\left(\tau-\tau_{0}\right) z_{0}+S\left(\tau-\tau_{0}\right) w_{0} \\
& \left.+\int_{\tau_{0}}^{\tau} S(\tau-v) \xi\left(v, z(v), \int_{\tau_{0}}^{v} \zeta(v, s, z(s)) d s\right) d v, \tau \in \tau_{0}, \Theta\right]
\end{aligned}
$$

is said to be the mild solution of equation (104).

Theorem 29. In this context, if

(a) the mapping $T: M \longrightarrow M$ defined for $\left.\tau \in \tau_{0}, \Theta\right]$ by

$$
\begin{aligned}
T z(\tau)= & C\left(\tau-\tau_{0}\right) z_{0}+S\left(\tau-\tau_{0}\right) w_{0} \\
& +\int_{\tau_{0}}^{\tau} S(\tau-v) \xi\left(v, z(v), \int_{\tau_{0}}^{v} \zeta(v, s, z(s)) d s\right) d v
\end{aligned}
$$

is continuous

(b) for $\tau, v \in\left[\tau_{0}, \Theta\right]$ and $z_{1}, z_{2}, t_{1}, t_{2} \in M$, there exist some constants $k_{1}>0, k_{2}>1$ such that

$$
\begin{gathered}
\left|\xi\left(\tau, z_{1}(\tau), t_{1}(\tau)\right)-\xi\left(\tau, z_{2}(\tau), t_{2}(\tau)\right)\right| \\
\leq k_{1}\left[\left|z_{1}(\tau)-z_{2}(\tau)\right|+\left|t_{1}(\tau)-t_{2}(\tau)\right|\right], \\
\left|\zeta\left(\tau, v, z_{1}(v)\right)-\zeta\left(\tau, v, z_{2}(v)\right)\right| \leq k_{2}\left[\left|z_{1}(v)-z_{2}(v)\right|\right]^{1 / 2} .
\end{gathered}
$$

(c) $N k_{1} \Theta^{2}\left[N k_{1}\left(1+k_{2} \Theta\right)+k_{2}\right]<1$ and $N k_{1}\left(1+k_{2} \Theta\right)>1$ then equation (104) has a unique mild solution on [ $\left.\tau_{0}, \Theta\right]$.

Proof. We notice that the mild solution of equation (104) is precisely a fixed point of the mapping $T$. For this reason, we check if the assumption of Theorem 12 holds. Considering the function $\alpha: M \times M \longrightarrow[0, \infty)$ defined as $\alpha(z, t)=1$ for any $z, t \in M$, we have that $T$ is $\alpha$-orbital admissible and there exists $z_{0} \in M$ such that $\alpha\left(z_{0}, T z_{0}\right)=1$. Moreover, for $z, t \in M$, 
we have

$$
\begin{aligned}
& d(T z, T t)=\sup _{\left.\tau \in \tau_{0}, \Theta\right]}|T z(\tau)-T t(\tau)| \\
& =\sup _{\left.\tau \in \tau_{0}, \Theta\right]} \mid \int_{\tau_{0}}^{\tau} S(\tau-v) \xi\left(v, z(v), \int_{\tau_{0}}^{v} \zeta(v, s, z(s)) d s\right) d v \\
& --\int_{\tau_{0}}^{\tau} S(\tau-v) \xi\left(v, t(v), \int_{\tau_{0}}^{v} \zeta(v, s, t(s)) d s\right) d v \mid \\
& \leq \sup _{\left.\tau \in \tau_{0}, \Theta\right]} \int_{\tau_{0}}^{\tau}|S(\tau-v)| \mid \xi\left(v, z(v), \int_{\tau_{0}}^{v} \zeta(v, s, z(s)) d s\right) \\
& -\xi\left(\left(v, t(v), \int_{\tau_{0}}^{v} \zeta(v, s, t(s)) d s\right) \mid d v\right. \\
& \leq N \sup _{\left.\tau \in \tau_{0}, \Theta\right]} \int_{\tau_{0}}^{\tau} k_{1}\left[|z(v)-t(v)|+\mid \int_{\tau_{0}}^{v} \zeta(v, s, z(s)) d s\right. \\
& \left.-\int_{\tau_{0}}^{v} \zeta(v, s, t(s)) d s \mid\right] d v \leq N \int_{\tau_{0}}^{\tau} k_{1}\left[\sup _{\left.\tau \in \tau_{0}, \Theta\right]} \mid z(v)\right. \\
& \left.-t(v)\left|+\int_{\tau_{0}}^{v} k_{2} \sup _{\left.\tau \in \tau_{0}, \Theta\right]}\right| z(s)-\left.t(s)\right|^{1 / 2} d s\right] d v \\
& =N k_{1}\left[d(z, t)\left(\tau-\tau_{0}\right)+\int_{\tau_{0}}^{\tau} k_{2}[d(z, t)]^{1 / 2}\left(v-\tau_{0}\right) d v\right] \\
& =N k_{1}\left[d(z, t)\left(\tau-\tau_{0}\right)+k_{2}[d(z, t)]^{1 / 2} \frac{\left(\tau-\tau_{0}\right)^{2}}{2}\right] \text {. }
\end{aligned}
$$

Analogically,

$$
\begin{aligned}
d\left(T^{2} z, T^{2} t\right)= & \sup _{\left.\tau \in \tau_{0}, \Theta\right]}\left|T^{2} z(\tau)-T^{2} t(\tau)\right|=\sup _{\left.\tau \in \tau_{0}, \Theta\right]}|T(T z(\tau))-T(T t(\tau))| \\
\leq & \sup _{\left.\tau \in \tau_{0}, \Theta\right]} \int_{\tau_{0}}^{\tau}|S(\tau-v)| \mid \xi\left(v, T z(v), \int_{\tau_{0}}^{v} \zeta(v, s, T z(s)) d s\right) \\
& \left.--\xi\left(v, T t(v), \int_{\tau_{0}}^{v} \zeta(v, s, T t(s)) d s\right)\right] d v \\
\leq & N \sup _{\left.\tau \in \tau_{0}, \Theta\right]} \int_{\tau_{0}}^{\tau} k_{1}\left[|T z(v)-T t(v)|+\mid \int_{\tau_{0}}^{v} \zeta(v, s, T z(s)) d s\right. \\
& \left.-\int_{\tau_{0}}^{v} \zeta(v, s, T t(s)) d s \mid\right] d v \leq N \int_{\tau_{0}}^{\tau} k_{1}\left[\sup _{\left.\tau \in \tau_{0}, \Theta\right] \mid} \mid T z(v)\right. \\
& \left.-T t(v)\left|+\int_{\tau_{0}}^{v} k_{2} \sup _{\left.\tau \in \tau_{0}, \Theta\right]}\right| z(s)-\left.t(s)\right|^{1 / 2} d s\right] d v \\
= & N k_{1}\left[d(T z, T t)\left(\tau-\tau_{0}\right)+\int_{\tau_{0}}^{\tau} k_{2}[d(T z, T t)]^{1 / 2}\left(v-\tau_{0}\right) d v\right] \\
= & N k_{1}\left[d(T z, T t)\left(\tau-\tau_{0}\right)+k_{2}[d(T z, T t)]^{1 / 2} \frac{\left(\tau-\tau_{0}\right)^{2}}{2}\right],
\end{aligned}
$$

and taking (109) into account, we have

$$
\begin{aligned}
d\left(T^{2} z, T^{2} t\right) \leq & N k_{1}\left[N k_{1}\left[d(z, t)\left(\tau-\tau_{0}\right)+k_{2}[d(z, t)]^{1 / 2} \frac{\left(\tau-\tau_{0}\right)^{2}}{2}\right]\left(\tau-\tau_{0}\right)\right. \\
& \left.+k_{2}[d(T z, T t)]^{1 / 2} \frac{\left(\tau-\tau_{0}\right)^{2}}{2}\right] \\
\leq & N k_{1}\left[N k_{1}[d(z, t)]^{1 / 2}\left[\Theta^{2}+k_{2} \frac{\Theta^{3}}{2}\right]+k_{2}[d(T z, T t)]^{1 / 2} \frac{\Theta^{2}}{2}\right] \\
= & \left.\left.\left(N k_{1} \Theta\right)^{2}\left(1+k_{2} \frac{\Theta}{2}\right) \cdot d(z, t)\right]^{1 / 2}+N k_{1} k_{2} \frac{\Theta^{2}}{2} \cdot d(T z, T t)\right]^{1 / 2} \\
\leq & \left.\left.\left(N k_{1} \Theta\right)^{2}\left(1+k_{2} \Theta\right) \cdot d(z, t)\right]^{1 / 2}+N k_{1} k_{2} \Theta^{2} \cdot d(T z, T t)\right]^{1 / 2} .
\end{aligned}
$$

If we denote $k=N k_{1} \Theta^{2}\left[N k_{1}\left(1+k_{2} \Theta\right)+k_{2}\right]<1$, choosing $a_{1}=\left(N k_{1} \Theta\right)^{2}\left(1+k_{2} \Theta\right) / k<1, a_{4}=N k_{1} \Theta^{2} k_{2} / k<1, a_{2}=$ $a_{3}=a_{5}=\delta=0$, and $\lambda=1 / 2$, we have that the relation (35) holds. Therefore, $T$ is a hybrid-interpolative ReichIstrățescu-type contraction, and from Theorem 12, it follows that the mapping $T$ has a fixed point or, equivalent, that equation (104) has a mild solution.

\section{Conclusions and Future Lines of Research}

In this paper, we have introduced a great family of contractions that we have called hybrid-interpolative ReichIstrățescu-type contractions in the setting of metric spaces. The main advantage of this family is the fact that its general contractivity condition can be particularized in several ways, depending on many parameters $\left(\lambda, k, a_{1}, a_{2}, a_{3}, a_{4}, a_{5}, \delta\right)$. Furthermore, such contractivity condition involves many distinct terms that can be either adding or multiplying between them (it depends on $\lambda$ ). In addition to this, we must highlight that the main contractivity condition makes use of the self-composition $T^{2}=T \circ T$, whose associated theorems used to be more general than the corresponding results by only using $T$.

For the study of the behavior of the Picard sequence that is used in the main results, an analysis of the convergence of certain sequences of nonnegative real terms that naturally appear in the proofs that we have introduced has been carried out. If the reader pays attention, some of these sequences repeatedly appear in the field of the fixed point theory. For example, the condition " $d\left(z_{n+1}, z_{n+2}\right) \leq \lambda d\left(z_{n}, z_{n+1}\right)$ for all $n \in \mathbb{N}$ ” appears in many demonstrations in this field of study. Therefore, the results of Section 3 have been of great help in the development of the main results.

In this setting, we have demonstrated some fixed point theorems that guarantee the existence and, in some cases, the uniqueness, of fixed points. To illustrate the power of the introduced results, we have shown several consequences of the main statements. We have also remarked how the proposed results are optimal with respect to the involved parameters; that is, when a result includes the hypothesis about $\sum_{i=1}^{5} a_{i}+\delta<1$, we have described an example in which the equality in the previous strict inequality leads to the invalidity of the corresponding result. 
Finally, we have taken advantage of the main contents of the manuscript in order to introduce a novel application in which, under very general conditions, we are able to deduce the existence of solution of a kind of integral equations.

Prospective work must be done in this line of research. On the one hand, further studies should be done on how to reformulate the contractivity condition in order to be able to develop fixed point theory in more general abstract metric spaces (for instance, $b$-metric spaces or fuzzy metric spaces). On the other hand, we propose studying the possible existence of fixed points for an operator $T$ such that

$$
\begin{gathered}
\alpha(z, t) d\left(T^{2} z, T^{2} t\right) \leq k \cdot \max \{d(z, t), d(z, T z), d \\
\left.\cdot(t, T t), d(T z, T t), d\left(T z, T^{2} z\right), d\left(T t, T^{2} t\right)\right\},
\end{gathered}
$$

for all distinct points $z, t \in \Omega$, where $\Omega$ is an appropriate subset of a metric space $(M, d)$.

\section{Data Availability}

No data were used to support this study.

\section{Conflicts of Interest}

The authors declare that they have no competing interests.

\section{Authors' Contributions}

All authors contributed equally and significantly to writing this article. All authors read and approved the final manuscript.

\section{Acknowledgments}

The authors thank their respective universities. A.F. Roldán López de Hierro is grateful to Ministerio de Ciencia e Innovación by Project PID2020-119478GB-I00 and to Program FEDER Andalucía 2014-2020 by Project A-FQM-170UGR20.

\section{References}

[1] D. W. Boyd and J. S. W. Wong, "On nonlinear contractions," Proceedings of the American Mathematical Society, vol. 20, no. 2, pp. 458-464, 1969.

[2] J. Caristi, "Fixed point theorems for mappings satisfying inwardness conditions," Transactions of the American Mathematical Society, vol. 215, pp. 241-251, 1976.

[3] S. K. Chatterjea, "Fixed point theorems," Comptes rendus de l'Academie bulgare des Sciences, vol. 25, pp. 727-730, 1972.

[4] G. E. Hardy and T. D. Rogers, "A generalization of a fixed point theorem of Reich," Canadian Mathematical Bulletin, vol. 16, pp. 201-206, 1973.

[5] R. Kannan, "Some results on fixed points," Bulletin of the Calcutta Mathematical Society, vol. 60, pp. 71-76, 1969.

[6] R. Kannan, "Some results on fixed points II," The American Mathematical Monthly, vol. 76, pp. 405-408, 1969.

[7] L. B. Ćirić, "A generalization of Banachs contraction principle," Proceedings of the American Mathematical Society, vol. 45, no. 2, pp. 267-273, 1974.
[8] M. Geraghty, "On contractive mappings," Proceedings of the American Mathematical Society, vol. 40, no. 2, pp. 604-608, 1973.

[9] A. Meir and E. Keeler, "A theorem on contraction mappings," Journal of Mathematical Analysis and Applications, vol. 28, pp. 326-329, 1969.

[10] B. Samet, C. Vetro, and P. Vetro, "Fixed point theorem for $\alpha$ $\psi$-contractive type mappings," Nonlinear Analysis, vol. 75, pp. 2154-2165, 2012.

[11] F. Khojasteh, S. Shukla, and S. Radenovic, "A new approach to the study of fixed point theory for simulation functions," Univerzitet $u$ Nišu, vol. 29, no. 6, pp. 1189-1194, 2015.

[12] M. A. Kutbi, A. Roldán, W. Sintunavarat, J. Martínez-Moreno, and C. Roldán, "F-closed sets and coupled fixed point theorems without the mixed monotone property," Journal of Fixed Point Theory and Applications, vol. 2013, no. 1, 2013.

[13] M. Jleli and B. Samet, "A generalized metric space and related fixed point theorems," Fixed Point Theory and Applications, vol. 2015, no. 1, 2015.

[14] M. Berzig and B. Samet, "An extension of coupled fixed point's concept in higher dimension and applications," Computers \& Mathematcs with Applications, vol. 63, no. 8, pp. 1319-1334, 2012.

[15] E. Karapnar, "Revisiting the Kannan type contractions via interpolation," Advances in the Theory of Nonlinear Analysis and its Applications, vol. 2, pp. 85-87, 2018.

[16] E. Karapnar, R. Agarwal, and H. Aydi, "Interpolative ReichRus-Ćirić type contractions on partial metric spaces," Mathematics, vol. 6, no. 11, 2018.

[17] H. Aydi, C.-M. Chen, and E. Karapnar, "Interpolative ĆirićReich-Rus type contractions via the Branciari distance," Mathematics, vol. 7, no. 1, p. 84, 2019.

[18] V. Istratescu, "Some fixed point theorems for convex contraction mappings and mappings with convex diminishing diameters (I)," Annali di Matematica Pura ed Applicata, vol. 130, pp. 89-104, 1982.

[19] V. Istratescu, "Some fixed point theorems for convex contraction mappings and mappings with convex diminishing diameters (II)," Annali di Matematica Pura ed Applicata, vol. 134, pp. 327-362, 1983.

[20] O. R. N. Samadi and E. Tohidi, "The spectral method for solving systems of Volterra integral equations," Journal of Applied Mathematics and Computing, vol. 40, no. 1-2, pp. 477-497, 2012.

[21] F. Mirzaee, S. Bimesl, and E. Tohidi, "Solving nonlinear fractional integro-differential equations of Volterra type using novel mathematical matrices," Journal of Computational and Nonlinear Dynamics, vol. 10, no. 6, 2015.

[22] E. Tohidi, M. M. Ezadkhah, and S. Shateyi, "Numerical solution of nonlinear fractional Volterra integro-differential equations via Bernoulli polynomials," Abstract and Applied Analysis, vol. 2014, Article ID 162896, 7 pages, 2014.

[23] E. Tohidi, "Taylor matrix method for solving linear twodimensional Fredholm integral equations with piecewise intervals," Computational and Applied Mathematics, vol. 34, no. 3, pp. 1117-1130, 2015.

[24] J. S. Nadja, O. R. N. Samadi, and E. Tohidi, "Numerical solution of two-dimensional Volterra integral equations by spectral Galerkin method," Journal of Applied Mathematics \& Bioinformatics, vol. 1, no. 2, pp. 159-174, 2011. 
[25] Y. Yang, E. Tohidi, X. Ma, and S. Kang, "Rigorous convergence analysis of Jacobi spectral Galerkin methods for Volterra integral equations with noncompact kernels," Journal of Computational and Applied Mathematics, vol. 366, 2020.

[26] Z. Asgari, F. Toutounian, E. Babolian, and E. Tohidi, "LSMR iterative method for solving one- and two-dimensional linear Fredholm integral equations," Computational and Applied Mathematics, vol. 38, no. 3, 2019.

[27] V. Berinde, "Approximating fixed points of weak contractions using the Picard iteration," Nonlinear Analysis Forum, vol. 9, pp. 43-53, 2004.

[28] I. A. Bakhtin, "The contraction mapping principle in quasimetric spaces," Journal of Functional Analysis, vol. 30, pp. 26-37, 1989.

[29] S. Czerwik, "Nonlinear set-valued contraction mappings in bmetric spaces," Atti del Seminario Matematico e Fisico dell'Universita di Modena, vol. 46, pp. 263-276, 1998. 